\title{
Tumor primario maligno en aurícula izquierda con invasión a válvula mitral, venas pulmonares y metástasis cerebral. Informe de un caso
}

\author{
Malignant primary tumor in left atrium with mitral valve invasion, pulmonary veins and \\ cerebral metastasis. A case report
}

Miguel Reynoso-Hermosillo', Jaime Sandoval-García1, Baltazar López-Rosales ${ }^{1}$, Antonio Esparza-Villarruel', José Ma Díaz-Sifuentes², David García-Martínez ${ }^{2}$ y Wenceslao G. Ángeles-Bueno ${ }^{3 *}$

${ }^{1}$ Servicio de Cirugía Cardiaca, Unidad Médica de Alta Especialidad; ²Unidad de Investigación Biomédica 02; ${ }^{3}$ Servicio de Cirugía Oncológica, Unidad Médica de Alta Especialidad. Hospital de Especialidades, Centro Médico Nacional de Occidente, Instituto Mexicano del Seguro Social, Guadalajara, Jalisco, México

\section{Resumen}

Introducción: La incidencia de tumores primarios cardiacos se calcula en 0.0017 a $0.28 \%$, de los cuales el $25 \%$ es maligno y ocupa principalmente cavidades derechas. Caso clínico: Paciente que inicia con síntomas neurológicos por metástasis cerebrales de tumor primario cardiaco en cavidades izquierdas, que infiltra válvula mitral y venas pulmonares. Se realiza resección tumoral con reemplazo valvular mitral y remodelamiento auricular con uso de orejuela izquierda. Conclusiones: Las técnicas de imagen son fundamentales para el diagnóstico de esta afección, ya que la mayor parte de los tumores cardiacos se muestra asintomática hasta etapas tardías.

Palabras Clave: Tumor primario cardiaco. Sarcoma intimal auricular. Metástasis cerebrales. Tratamiento quirúrgico.

\begin{abstract}
Introduction: The incidence of cardiac primary tumors is estimated to be $0.0017-0.28 \%$, of which $25 \%$ are malignant, occupying mainly right cavities. Clinical case: Patient who present with neurological symptoms due to brain metastasis of primary cardiac tumor in the left atrium, infiltrating the mitral valve and pulmonary veins. Tumor resection was performed with mitral valve replacement and atrial re-modeling with the use of the left appendage. Conclusions: Imaging techniques are fundamental for the diagnosis of this pathology, since the majority of cardiac tumors are asymptomatic until late stages.
\end{abstract}

Key Words: Cardiac primary tumor. Primary intimal sarcoma. Brain metastasis. Surgical management.

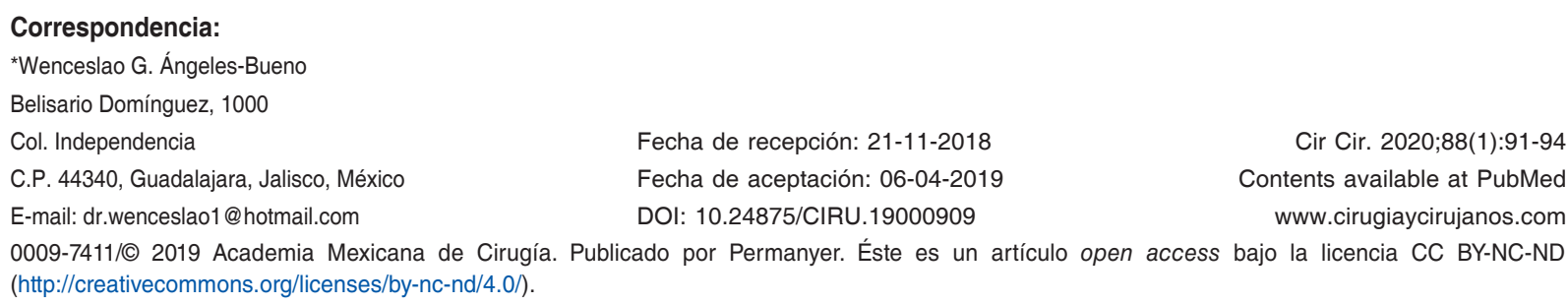

Fecha de recepción: 21-11-2018

Fecha de aceptación: 06-04-2019

C.P. 44340 , Guadalajara, Jalisco, México

E-mail: dr.wenceslao1@ hotmail.com

DOI: $10.24875 / C I R U .19000909$

0009-7411/@ 2019 Academia Mexicana de Cirugía. Publicado por Permanyer. Éste es un artículo open access bajo la licencia CC BY-NC-ND (http://creativecommons.org/licenses/by-nc-nd/4.0/). 


\section{Introducción}

Los tumores primarios cardiacos malignos son neoplasias extremadamente raras de histopatología variada. Representan el $0.008 \%$ de los casos de cáncer; el más frecuente es el sarcoma y de él el subtipo angiosarcoma ${ }^{1}$.

La presentación de los síntomas ocurre de una manera muy variada, lo cual depende del tamaño y la localización. Por lo tanto, para proveer información morfológica de un tumor y sus consecuencias funcionales son necesarios los estudios de imagen ${ }^{2}$. Los sarcomas de la aurícula izquierda tienden a ser más sólidos y menos infiltrativos ${ }^{3}$.

El tratamiento de elección es la resección completa, con reconstrucción o reemplazo valvular de ser necesario para mantener márgenes libres. En casos seleccionados, el trasplante cardiaco se considera el tratamiento final, una vez descartada la presencia de metástasis ${ }^{4}$. En caso de metástasis o si la resección marginal no es posible, el tratamiento debe complementarse con radioterapia. $\mathrm{Y}$ aunque se ha demostrado que la quimioterapia no aumenta la sobrevida, si reduce el riesgo de episodios adversos y, por ende, se recomienda emplearla en presencia de metástasis, resección incompleta o en pacientes no elegibles para intervención quirúrgica ${ }^{5}$.

\section{Caso clínico}

Paciente masculino de 34 años de edad, alérgico a las sulfamidas, sin más antecedentes patológicos ni comorbilidades. Inicia su padecimiento en el mes de septiembre de 2017 y refiere cefalea hemicraneal izquierda, que cede con analgésicos; dos meses después se intensifica y se acompaña de vértigo que llega a ser incapacitante, motivo por el cual acude al Hospital General Regional.

Se inició abordaje diagnóstico con tomografía axial computarizada (TC) y resonancia magnética nuclear (RM) (Fig. 1) en las que se observa una tumoración parietooccipital izquierda, lesión quística con reforzamiento en halo hiperintenso. Se lo envía al Servicio de Neurocirugía y allí se realiza craniectomía, que identifica un tumor parietal parasagital con cápsula engrosada que contiene líquido amarillo oleoso.

En el estudio histológico se observó un tumor de células de Sertoli poco diferenciado, por lo que se realizó una TC que delineó un tumor mediastínico. Con posterioridad se efectuó ecosonograma transtorácico

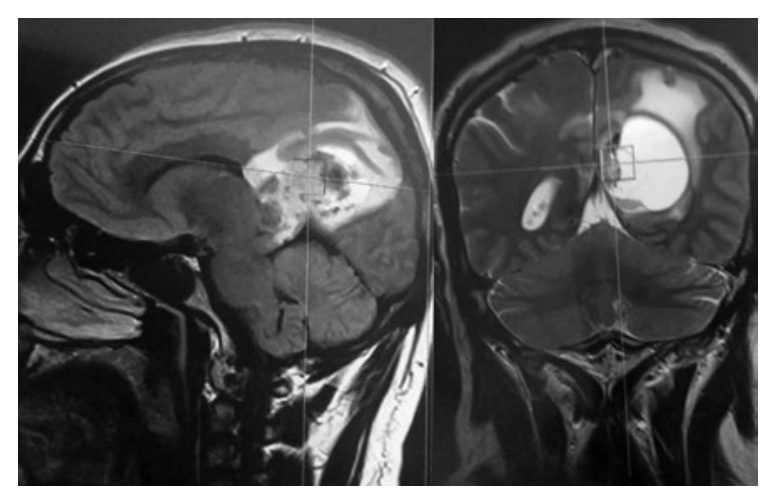

Figura 1. Resonancia magnética en la que se demuestra la tumoración cerebral.

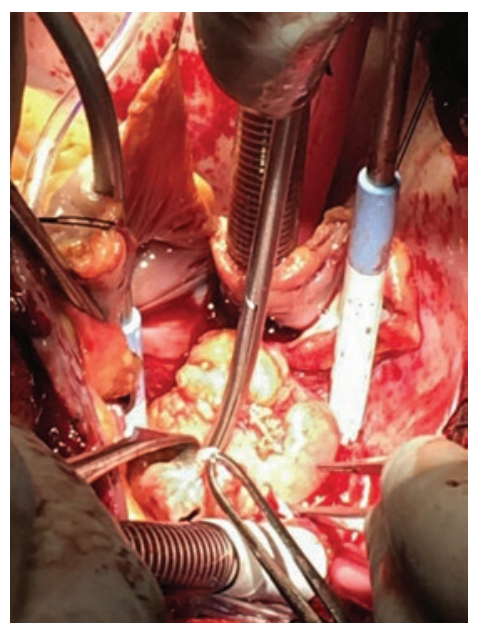

Figura 2. Tumor cardiaco auricular izquierdo.

(ECOTT), que documentó un tumor en el techo de la aurícula izquierda de 17 x $19 \mathrm{~mm}$, de base amplia; se solicitó además RM que encontró hallazgos de tumor en la aurícula izquierda de $34 \times 30 \times 37 \mathrm{~mm}$ que ocupaba el $50 \%$ de la aurícula, con infiltración a la vena pulmonar inferior derecha.

Se decidió practicar resección tumoral cardiaca y reemplazo valvular mitral; los hallazgos notificados fueron: tumor en el techo auricular izquierdo que comprometía anillo mitral y valva anterior de la válvula mitral, así como vena pulmonar inferior derecha de aspecto sésil, indurado y de características malignas (Fig. 2).

Se realizó un procedimiento bajo anestesia general balanceada, vigilancia invasiva de clase II, con incisión medioesternal, exposición cardiaca y protocolo para derivación cardiopulmonar con doble cava; se llevó a cabo abordaje transeptal alto que se amplió con sección de la vena cava superior (Fig. 3). A continuación se realizó una resección tumoral extensa que sacrificó el techo auricular izquierdo, la válvula y el anillo mitral, y se efectuó reemplazo de 


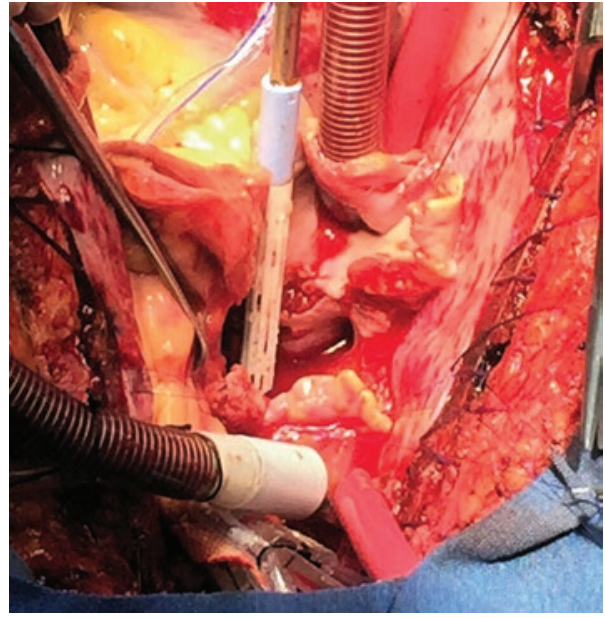

Figura 3. Abordaje quirúrgico.

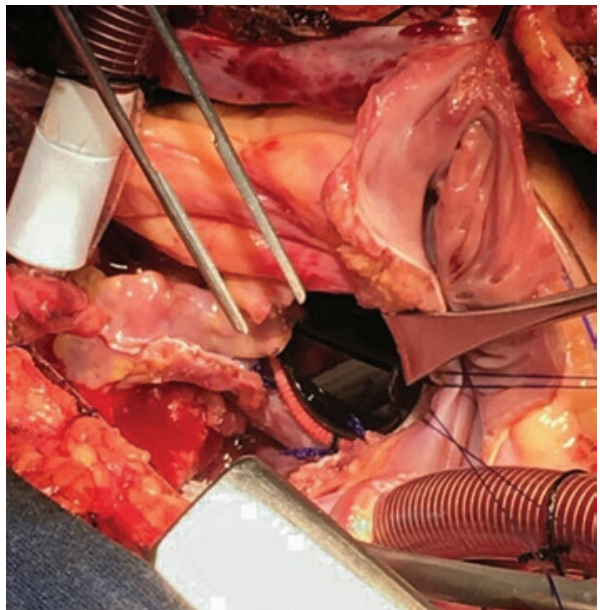

Figura 4. Colocación de una prótesis mitral mecánica de $31 \mathrm{~mm}$.

la válvula mitral nativa invadida por una válvula mecánica $(31 \mathrm{~mm})$ (Fig. 4) y reconstrucción auricular izquierda. Se llevaron a cabo plastia mediante la orejuela ipsolateral, anastomosis de vena cava superior, cierre del septum interauricular y cierre auricular derecho. Salida de bomba en el primer intento en ritmo nodal apoyado con marcapasos temporal y después pasó a ritmo sinusal; se solicitó ECOTT transquirúrgico en el cual se observó la ausencia de tumor, así como la adecuada movilidad valvular protésica; se colocaron drenajes mediastínicos y se completó el procedimiento.

Pasó a la unidad de cuidados intensivos posquirúrgicos donde presentó neumonía, insuficiencia hepática aguda y lesión renal aguda, con resolución adecuada. Se egresó a piso de cardiocirugía, para continuar tratamiento y seguimiento: la TC de control reveló cambios posoperatorios en la pared anterior y superior de la aurícula izquierda sin observar tejido residual tumoral, cavidades cardiacas de tamaño normal, las cuatro

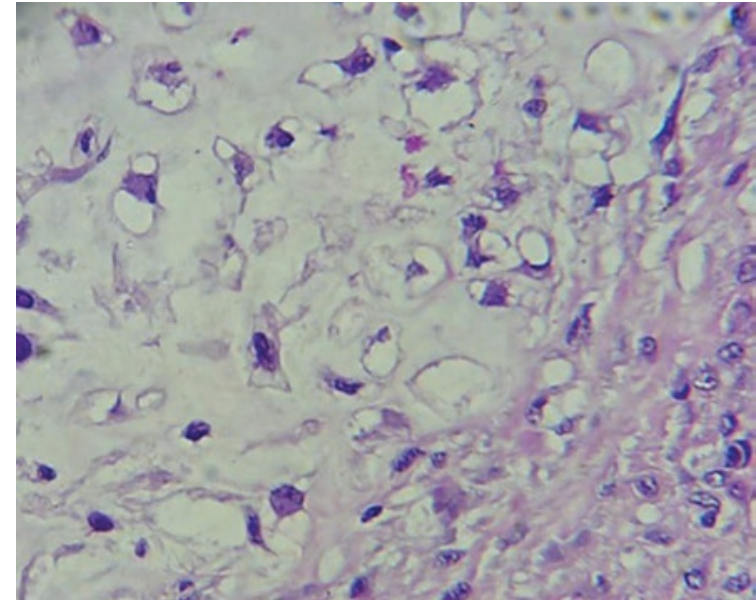

Figura 5. Zonas de metaplasia condroide; se observan células de núcleos irregulares inmersos en matriz condroide $(40 x$, tinción de hematoxilina-eosina).

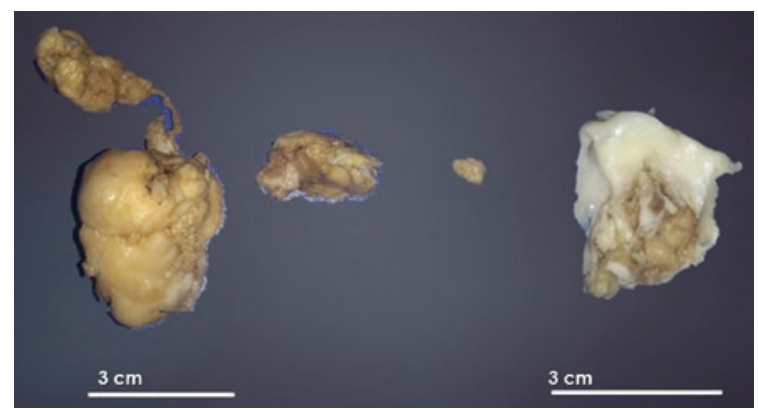

Figura 6. Plano macroscópico: tumoración de aspecto polipoide irregular, color café claro. A la derecha se observa un segmento de la válvula afectado por la proliferación de la tumoración.

venas pulmonares permeables sin defectos de llenado, ECOTT sin evidencia de lesión tumoral con válvula protésica normofuncional y fracción de expulsión del ventrículo izquierdo preservada. Se indicó el egreso a domicilio.

\section{Discusión}

Los tumores primarios cardiacos malignos son neoplasias extremadamente raras de histopatología variada. El tipo más frecuente es el sarcoma con subtipo intimal ${ }^{6}$, el mismo que presentó el paciente (sarcoma intimal auricular primario de alto grado) (Figs. 5 y 6), cuya característica patológica es la ampliación del gen MDM2?.

En este país existen dos series quirúrgicas publicadas de casos de tumores cardiacos primarios, con una prevalencia de $0.73 \%$ y $0.16 \%$, y se ha encontrado estirpe maligna en el $12.5 \%$ y $16 \%$ respectivamente; el sarcoma es el tipo predominante ${ }^{8,9}$. Se han descrito informes de casos aislados en los que la 
presentación clínica incluye síntomas cardiacos, además de raros casos de linfomas e histiocitomas fibrosos malignos ${ }^{10-13}$.

Los síntomas de esta anomalía son muy variados y se presentan sobre todo insuficiencia cardiaca congestiva por obstrucción, embolización, síntomas constitucionales y arritmias, aunque en este caso el paciente inició con signos neurológicos por metástasis cerebral, a pesar de que los sarcomas de la aurícula izquierda emiten metástasis de forma tardía, ya que tienden a ser más sólidos y menos infiltrativos que los del lado derecho ${ }^{3}$.

Debido a su difícil diagnóstico clínico, los estudios de imagen pueden utilizarse para proveer información morfológica de un tumor cardiaco y sus consecuencias funcionales. El principal objetivo es caracterizar la localización del tumor, tamaño y vascularidad y consecuencias hemodinámicas y valorar la infiltración. Un ECOTT debe efectuarse en todos los pacientes y puede usarse para definir la relación con otras estructuras y el sitio exacto de fijación. Mientras que la RM ayuda a definir grado, vascularidad e infiltración del tumor, también permite junto con el ecocardiograma una mejor perspectiva para el diagnóstico y ayuda a diferenciar entre tumores malignos y no malignos ${ }^{2}$. En este caso informado, la TC ayudó a identificar el sitio de tumoración en la región mediastínica para luego identificar el sitio de dicha tumoración mediante ECOTT.

En el tratamiento para los tumores malignos confinados al corazón, que no tienen infiltración, se puede realizar una resección tumoral compleja, por ejemplo resección total de cavidades derechas, y asegurar una circulación pulmonar de tipo Fontan. Se puede practicar una resección ex situs en caso de que el tumor envuelva la pared posterior de la aurícula izquierda o los grandes vasos. Cuando el tumor envuelve por completo el lado izquierdo, sin evidencia de metástasis, es plausible utilizar un corazón artificial total ${ }^{4}$. En casos seleccionados, el trasplante cardiaco se considera el tratamiento final, una vez descartada la presencia de metástasis.

Con este paciente, la operación se practicó con éxito y se consiguió una resección completa con márgenes adecuados comprobados por estudio de histopatología.

La sobrevida informada para los pacientes con tumores cardiacos es aún escasa. La media notificada es de 6 a 18 meses, que es significativamente mayor cuando se logra la resección completa en comparación con aquellos que no tienen metástasis en su presentación $n^{6}$. Los autores llevaron un seguimiento en la consulta externa de nueve meses sin evidencia de recurrencia.

\section{Conflicto de intereses}

Los autores declaran no tener conflicto de intereses.

\section{Financiamiento}

Los autores declaran que este artículo se realizó con recursos propios de la institución.

\section{Responsabilidades éticas}

Protección de personas y animales. Los autores declaran que para esta investigación no se han realizado experimentos en seres humanos ni en animales.

Confidencialidad de los datos. Los autores declaran que en este artículo no aparecen datos de pacientes.

Derecho a la privacidad y consentimiento informado. Los autores han obtenido el consentimiento informado de los pacientes y/o sujetos referidos en el artículo. Este documento obra en poder del autor de correspondencia.

\section{Bibliografía}

1. Oliveira GH, Al-Kindi SG, Hoimes C, Park SJ. Characteristics and survival of malignant cardiac tumors: a 40 -year analysis of $>500$ patients. Circulation. 2015;132:2395-402.

2. Bhattacharyya S, Khattar RS, Gujral DM, Senior R. Cardiac tumors: the role of cardiovascular imaging. Expert Rev Cardiovasc Ther. 2014;12:37-43.

3. Leja MJ, Shah DJ, Reardon MJ. Primary cardiac tumors. Tex Heart Inst J. 2011;38:261-2.

4. Hoffmeier A, Sindermann JR, Scheld HH, Martens S. Cardiac tumorsdiagnosis and surgical treatment. Dtsch Arztebl Int. 2014;111:205-116.

5. Lestuzzi C, De Paoli A, Baresic T, Miolo G, Buonadonna A. Malignant cardiac tumors: diagnosis and treatment. Future Cardiol. 2015;11:485-500.

6. Lamba G, Frishman WH. Cardiac and pericardial tumors. Cardiol Rev. 2012;20:237-52.

7. Neuville A, Collin F, Bruneval P, Parrens M, Thivolet F, Gómez-Brouchet A, et al. Intimal sarcoma is the most frequent primary cardiac sarcoma: clinicopathologic and molecular retrospective analysis of 100 primary cardiac sarcomas. Am J Surg Pathol. 2014;38:461-9.

8. Villalpando E, Pérez FM, Cortés AO, Y Cols. Frecuencia de tumores cardiacos y su correlación histopatológica. Cir Ciruj 2006;74:183-187.

9. Alfaro F, Careaga G, Valer G. Tumores cardiacos. Experiencia en 16 años en el hospital de cardiología del Centro Médico Nacional Siglo XXI. Cir Ciru 2003; 71:179-185.

10. Urías R, de la Cerda G, Cuevas A. Histiocitoma fibroso maligno intracardiaco. Reporte de un caso. Cirugía y Cirujanos. 2012;80(2):182-185.

11. García G, Dolores B, Zaunbos V. Sarcoma cardiaco primario: reporte de un caso y revisión de literatura actual. Rev Mex Cardiol. 2010;21(3):148-153.

12. Galván L, García E, Verdín R, et al. Tumores malignos cardiacos. Rev Mex Cardiol. 2008;19(3):138-144.

13. Campos A, Patiño E, Sánchez A. Linfoma cardiaco primario, una presentación extremadamente rara. Arch Cardiol Méx. 2016;86(1):94-96. 